\title{
Effectiveness of Interventions to Increase Colorectal Cancer Screening Among American Indians and Alaska Natives
}

\author{
Donald Haverkamp, $\mathrm{MPH}^{1}$; Kevin English, $\mathrm{DrPH}^{2}$; Jasmine Jacobs-Wingo, $\mathrm{MPH}^{1}$; \\ Amanda Tjemsland, BA ${ }^{1}$; David Espey, MD $^{1}$
}

\begin{abstract}
Accessible Version: www.cdc.gov/pcd/issues/2020/20_0049.htm
Suggested citation for this article: Haverkamp D, English K, Jacobs-Wingo J, Tjemsland A, Espey D. Effectiveness of Interventions to Increase Colorectal Cancer Screening Among American Indians and Alaska Natives. Prev Chronic Dis 2020; 17:200049. DOI: https://doi.org/10.5888/pcd17.200049.
\end{abstract}

\section{PEER REVIEWED}

\section{Summary}

What is already known about this topic?

Reducing client and structural barriers can result in greater participation in colorectal cancer screening, when stool-based tests are used.

What is added by this report?

Direct mailing of fecal immunochemical test kits was an effective strategy to increase colorectal cancer screening participation at rural, tribally operated health care facilities.

\section{What are the implications for public health practice?}

Stool-based testing is often the most accessible colorectal cancer screening option at rural, tribally run health care facilities. Direct mailing of fecal immunochemical tests may increase colorectal screening at health care facilities that serve American Indian and Alaska Native populations.

\section{Abstract}

\section{Introduction}

Screening rates for colorectal cancer are low in many American Indian and Alaska Native (AI/AN) communities. Direct mailing of a fecal immunochemical test (FIT) kit can address patient and structural barriers to screening. Our objective was to determine if such an evidence-based intervention could increase colorectal cancer screening among $\mathrm{AI} / \mathrm{AN}$ populations.

\section{Methods}

We recruited study participants from 3 tribally operated health care facilities and randomly assigned them to 1 of 3 study groups: 1) usual care, 2) mailing of FIT kits, and 3) mailing of FIT kits plus follow-up outreach by telephone and/or home visit from an American Indian Community Health Representative (CHR).

\section{Results}

Among participants who received usual care, $6.4 \%$ returned completed FIT kits. Among participants who were mailed FIT kits without outreach, $16.9 \%$ returned the kits - a significant increase over usual care $(P<.01)$. Among participants who received mailed FIT kits plus CHR outreach, $18.8 \%$ returned kits, which was also a significant increase over usual care $(P<.01)$ but not a significant increase compared with the mailed FIT kit-only group $(P=.44)$. Of 165 participants who returned FIT kits during the study, $39(23.6 \%)$ had a positive result and were referred for colonoscopy of which $23(59.0 \%)$ completed the colonoscopy. Twelve participants who completed a colonoscopy had polyps, and 1 was diagnosed with colorectal cancer.

\section{Conclusion}

Direct mailing of FIT kits to eligible community members may be a useful, population-based strategy to increase colorectal cancer screening among $\mathrm{AI} / \mathrm{AN}$ people.

\section{Introduction}

Colorectal cancer (CRC) is the second leading cause of death from cancer among American Indian and Alaska Native (AI/AN) men and third among AI/AN women (1). Although screening has been shown to reduce death rates, the percentage of people up to date with CRC screening is low in many $\mathrm{AI} / \mathrm{AN}$ communities. Less than half (48.4\%) of AI/AN adults aged 50 to 75 were up to date with CRC screening in 2015 (2). 
The US Preventive Services Task Force (USPSTF) recommends stool-based tests and direct visualization tests (colonoscopy, flexible sigmoidoscopy, or virtual colonoscopy) for CRC screening. (3). In health care systems with limited capacity to provide directvisualization screening tests, stool-based tests such as highsensitivity, guaiac-based fecal occult blood tests (FOBT) and fecal immunochemical tests (FIT) are often the most accessible options for CRC screening. However, various patient and structural barriers exist to completing FOBT and FIT: geographic isolation, lack of a regular health care provider, failure of providers to recommend screening, lack of clinical tracking and reminder systems, lack of transportation, embarrassment, privacy concerns, distrust of the health care system, and insufficient knowledge about CRC, its risk factors, and screening recommendations (4). Many of these barriers can be mitigated. According to the Community Preventive Services Task Force, there is sufficient evidence that using patient reminders and small media (eg, letters, pamphlets, brochures, flyers) can increase CRC screening with stool tests (5). Reducing structural barriers (eg, eliminating or simplifying administrative procedures required for CRC screening, reducing time or distance for screening services) is also an effective way to increase the use of stool tests (6). Direct mailing of FOBT or FIT is an approach that can address both patient and structural barriers. Mailing FOBT or FIT kits to patients and providing outreach through telephone calls and home visits can reduce patient and structural barriers, and both have been shown to be effective strategies to improve participation in CRC screening in various underserved populations (7-10). The objective of our study was to determine if such evidence-based interventions could also lead to increased $\mathrm{CRC}$ screening among rural $\mathrm{AI} / \mathrm{AN}$ populations.

\section{Methods}

\section{Participant recruitment}

We recruited 3 tribally operated health care facilities with which we had a previous working relationship to participate in our study. The selected facilities were in different tribal communities. At each facility, the clinic director used Resource and Patient Management System Query Manager (11) to generate a list of active clinic users (people who had obtained services at least once in the past 3 years), were aged 50 to 75 , were not up to date with CRC screening per USPSTF criteria at the time the study began (had not had an FOBT or FIT in the past year, flexible sigmoidoscopy in the past 5 years combined with FOBT or FIT in the past three years, or colonoscopy in the past 10 years) (12), and had no history of CRC or total colectomy. These criteria were met by 1,288 people. Our study was approved by institutional review boards of the Centers for Disease Control and Prevention (CDC) and the 3 participating tribal health care facilities.
Study design. At each facility, study participants were randomly assigned to 1 of 3 study groups: group 1 (the control or usual care group), in which participants visited the clinic with the same frequency as they would outside study conditions and received a FIT kit only if a provider recommended one; group 2 (mailing alone), in which participants were mailed FIT kits (Polymedco OC-Light), completion instructions (in English), a letter (in English) notifying them that they were due for CRC screening, and a prestamped, pre-addressed envelope for returning their completed FIT kit; and group 3 (mailing plus outreach), in which participants were mailed the same materials as group 2 and also received telephone and/or home visit follow-up from an American Indian Community Health Representative (CHR) if they did not return the completed test (Figure 1). At 2 study facilities, we randomized 133 CRC screening-eligible participants to each of the 3 groups, and included the remaining 205 screening-eligible patients at these 2 facilities in the usual-care group (124 people at one clinic and 81 people at the other clinic) (Table). Because 1 study clinic had a smaller patient population, we randomized all 285 CRC screeningeligible people at that facility equally among the 3 study groups (95 in each group). Providers were blinded to their patients' involvement in the study or study group. We hypothesized that the percentage of eligible persons completing FIT in each of the 2 intervention groups would be significantly higher than the percentage completing FIT in the usual care group (group 1), and that the percentage completing FIT in the mail-out plus outreach group (group 3) would be significantly higher than the percentage completing FIT in the mail-out alone group (group 2).

\footnotetext{
The opinions expressed by authors contributing to this journal do not necessarily reflect the opinions of the U.S. Department of Health and Human Services,
} the Public Health Service, the Centers for Disease Control and Prevention, or the authors' affiliated institutions. 


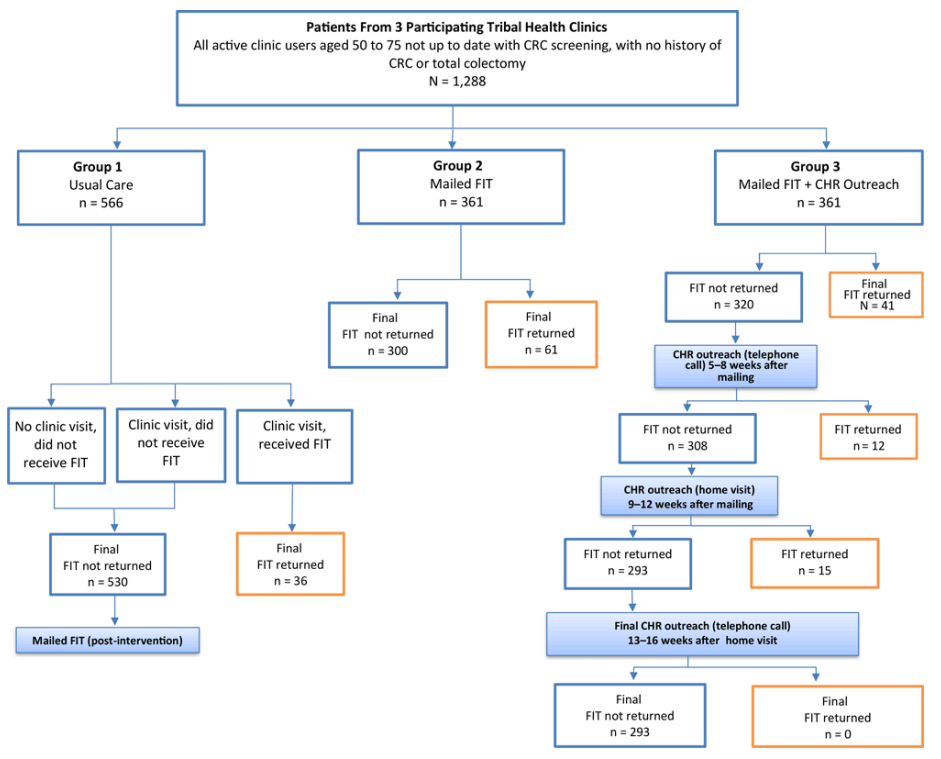

Figure 1. Participant selection, randomization, and outcomes in 3 study groups, intervention to increase colorectal cancer screening among American Indian and Alaska Native people $(N=1,288)$ served by 3 tribally operated health care clinics, April to November, 2014. Group 1, usual care, consisted of people who either did not visit the clinic, visited the clinic and did not receive a fecal immunochemical test (FIT) kit, or visited and received a FIT kit and instructions to complete at home. Group 2 participants received a FIT kit and completion instructions by mail. Group 3 participants received a mailed FIT kit and instructions, and nonrespondents received follow-up from a tribal community health representative after 4 weeks (by telephone), after 8 weeks (by home visit), and after 12 weeks by telephone. Abbreviations: CHR, community health representative; FIT, fecal immunochemical test.

Intervention design. We educated CHRs from each facility about CRC screening recommendations and our intervention protocol. We also informed clinic administrators and staff at each facility about the study. In April 2014, we mailed FIT kits, instructions for completion, an official letter from the clinic, and prestamped envelopes to participants in the intervention groups (groups 2 and 3). In August 2014, we mailed a follow-up letter to nonrespondents in groups 2 and 3 who had not yet returned kits, encouraging them to do so. The intervention period for all study groups was April 2014 through November 2014.

The outreach intervention protocol (group 3) instructed CHRs to make up to 5 attempts to contact by telephone all participants who had not returned their FIT kits within 4 weeks of the mailing; CHRs were to make up to 3 attempts to conduct a home visit to those who had not returned their FIT within 8 weeks, and up to 5 attempts to contact nonrespondents by telephone who had not returned the kits by the end of week 12 (Figure 1). If at first attempt a participant's telephone number was found to be disconnected or incorrect, CHRs were to visit that participant's home as the initial outreach activity. As part of their outreach, CHRs were to confirm that the participant received the mailed FIT kit (and provide another FIT kit if the participant did not receive the first), discuss the importance of CRC screening, review procedures for completing the FIT kit, encourage the participant to complete the FIT kit, answer questions, and offer to transport the completed FIT kit to the clinic laboratory.

Data tracking procedures. We created 2 databases to track results: 1 for laboratory staff to collect patient contact information and demographics, how and when FIT kits were disseminated and returned, and test results and another for CHRs to gather patient contact information and demographics, outreach type (telephone call or home visit), and other outreach details. Only clinic directors (or their designees), laboratory directors, and CHRs had access to the databases.

On-site clinic laboratories processed all completed FIT kits. Laboratory staff recorded FIT results in the participant tracking database and patient medical charts. Per standard operating procedures (3), clinic providers were instructed to refer any participant with a positive FIT result for colonoscopy.

Data analysis. Both the laboratory and CHR tracking databases were de-identified after the study intervention period, and the data files from all 3 facilities were merged. We used SPSS 22 (IBM Corp) software to perform Pearson $\chi^{2}$ testing to determine significant differences $(P<.05)$ in FIT completion between study groups.

\section{Results}

The mean age of the 1,288 study participants was 60 , half were aged 50 to 59 , and $52 \%$ were women. (Table). Overall, $12.8 \%$ $(165 / 1,288)$ returned a completed FIT kit to their clinic, and FIT completion did not differ by sex $(P=.52)$. The proportion who returned FIT kits increased with age: $10.8 \%$ (70/648) aged 50 to 59 , $13.6 \%(66 / 484)$ aged 60 to 69 , and $18.8 \%(29 / 154)$ aged 70 to 75 $(P=.02)$. Most who completed FIT kits hand delivered them to the clinic $(83.0 \%)$, whereas $16.4 \%$ used the pre-stamped, preaddressed envelope to return the kit by mail. Only one completed FIT kit $(0.6 \%)$ was delivered to the clinic by a CHR.

The percentage of returned FIT kits varied by study group (Figure 2 ). Among the participants who received usual care (group 1), $6.4 \%(36 / 566)$ completed their FIT kits at home and returned them to the clinic. In group 2 (mailing alone), 16.9\% (61/361) returned the FIT kits, a significant increase over group $1(P<.01)$. In group 3 (mailing plus outreach), 18.8\% (68/361) returned FIT kits to the clinic, a significant increase over group $1(P<.01)$, but not group $2(P=.44)$ (Figure 2$)$. Among those who returned a FIT kit, more women than men returned them in group $3(50.0 \%$ vs $31.6 \%)$ and more men than women in group $2(43.0 \%$ vs $31.4 \%)(P=.06)$.

The opinions expressed by authors contributing to this journal do not necessarily reflect the opinions of the U.S. Department of Health and Human Services, the Public Health Service, the Centers for Disease Control and Prevention, or the authors' affiliated institutions. 


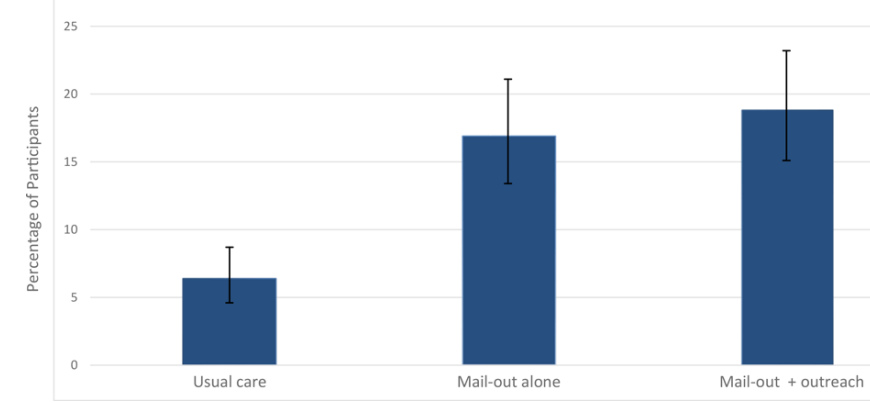

Figure 2. Percentage of participants who completed the fecal immunochemical test, by intervention group. Brackets indicate confidence intervals.

Among all group 3 participants, 11.4\% (41/361) returned their FIT kit during the 4-week period before any CHR outreach began. After receiving a single round of $\mathrm{CHR}$ outreach, an additional $3.3 \%(12 / 361)$ returned their FIT kit. Following a second round of CHR outreach, another $4.2 \%$ (15/361) returned their FIT kit. No additional FIT kits were returned to the clinics among participants who received a third round of outreach (Figure 1). Because of delays in the implementation of the CHR intervention, varying rounds of outreach were still being conducted with participants in the months following the mailing of a reminder letter at the end of the intervention timeframe. Following the reminder letter mailing, 17 people in group 3 and 13 in group 2 returned their FIT kits. Of these 17 from group 3,10 also received CHR outreach during that time. Overall, 51.5\% (35/68) of group 3 participants who returned their FIT kits received outreach of some kind (telephone call and/ or home visit) during the intervention period, including a few who received telephone call or home visit outreach even though they had already returned their FIT kit. Of the 293 participants in group 3 who did not return FIT kits, 76.8\% (225/293) received outreach of some kind (telephone call and/or home visit) during the intervention period.

Of the 165 FIT kits returned, 39 had a positive result; all 39 were referred for colonoscopy, and 23 of the 39 completed the colonoscopy. Results of those colonoscopies showed that 12 participants had polyps, and 1 participant was diagnosed with CRC.

\section{Discussion}

Our study showed that a significant increase in CRC screening participation is possible in AI/AN communities by mailing FIT kits and instructions to eligible community members and providing easy options for returning the kits to the clinic. The addition of telephone and home visit outreach following the FIT mailing also increased screening compared with the usual care group in our study, but not significantly beyond the level attained by only mailing FIT kits. Results similar to ours were reported by Coronado et al (8), with post-intervention CRC screening rates of $26 \%$ among Hispanic patients who received mailed FOBT only and $31 \%$ in the group that received mailed FOBT plus telephone call and home visit outreach; both results were significantly higher than the $2 \%$ screened in the usual care group, but not significantly different from one another. Another study demonstrated that the addition of telephone calls to encourage screening and to address barriers did not result in increased FIT completion compared with just mailing a FIT kit with printed educational materials (13). In contrast, Walsh et al (7) reported that self-reported FOBT screening rates among Latinos and Vietnamese patients at 1-year follow up increased by $7.8 \%$ in the usual care group, $15.1 \%$ in an FOBT mailing and brochure group, and $25.1 \%$ in a mailing, brochure, and telephone counseling group. The differences were significant between usual care and each intervention and between the 2 intervention groups.

One possible reason that our study's CHR outreach failed to significantly boost the FIT return percentage compared with mailing alone was the lack of the CHR intervention among many group 3 participants. Of those in group 3 who did not return their FIT, nearly 1 in 4 did not receive any outreach. This most likely occurred because of staff turnover during the study period and competing CHR job duties that limited the time available to implement the outreach as specified in the study protocol. In some instances, CHRs could not reach participants because of incorrect phone numbers or addresses - a common barrier to conducting community outreach. In a similar study by Jean-Jacques et al (14), $23 \%$ of participants had incorrect or nonfunctional telephone numbers. Lasser et al (15) reported that of those eligible for patient navigation, $25 \%$ could not be contacted after 8 to 11 telephone call attempts. When a tribal facility or health system chooses to use CHRs to assist with cancer screening, CHRs need to have designated time to focus on this task. Patient navigators hired in 1 facility in Alaska specifically to assist with CRC screening efforts dramatically increased the number of CRC patients' first-degree relatives who completed screening (16). Future studies could seek to determine how much outreach is appropriate before reaching saturation. In our study, no additional FIT kits were returned after the second round of outreach.

Even though our study showed a significant increase in return of FIT kits from participants who received mailed kits compared with usual care, the percentage of mailed kits that were returned in both intervention groups combined (17.9\%) was still low. Many reasons have been identified for nonresponse to a direct mailing of stool test kits, including fear of results, cost of follow-up colono-

\footnotetext{
The opinions expressed by authors contributing to this journal do not necessarily reflect the opinions of the U.S. Department of Health and Human Services, the Public Health Service, the Centers for Disease Control and Prevention, or the authors' affiliated institutions.
} 
scopy, not having received the mailed test, concerns about mailing fecal matter, and forgetfulness (17). Cultural barriers in AI/AN communities, such as medical mistrust, may also be a factor (4). Most participants in our study hand-delivered their completed FIT to the clinic instead of using the mailing envelope. Concern over mailing fecal material could be investigated further in this population. Additionally, some study participants may have had a language barrier. In our study, all written information with the FIT kit was in English. One alternative is to send out wordless instructions (eg, images/photographs) for completing the mailed FIT kit (18).

When stratified by age, our results showed that the percentage of returned FIT kits was highest at older ages. In the overall US population, CRC screening has been shown to be about $18 \%$ at age 50 , increasing to $28 \%$ by age 51 (19). AI/AN people are less likely than other racial/ethnic groups to initiate screening at the recommended aged of 50 (20) and are more likely to be diagnosed with $\mathrm{CRC}$ at ages younger than 50, compared with non-Hispanic white people (21). Providers serving AI/AN populations need to ensure that their patients begin screening at the appropriate age and continue screening at the correct intervals, depending on their chosen method of screening and CRC risk level.

A large percentage of participants who returned FIT kits in our study $(24 \%)$ had a positive FIT result. In a study by Hubbard et al (22), the risk of having a false-positive result from an FOBT was significantly greater among AI/AN than white patients. The greater risk of false-positive results among $\mathrm{AI} / \mathrm{AN}$ populations could be a result of using FOBT for both symptomatic and asymptomatic patients. Any facility considering implementing population-based screening with FOBT or FIT in a population that has not been screened previously may need to prepare for a higher-thanexpected proportion of positive test results and secure a facility that can perform the necessary follow-up colonoscopies.

Screening with FOBT or FIT reduces mortality from CRC only if patients with positive results undergo a follow-up colonoscopy. In our study, $41 \%$ of those with positive FIT results did not receive a follow-up colonoscopy. Several documented reasons for not completing colonoscopy are competing health concerns, failure to respond to follow-up outreach telephone calls and mailings, refusal, moving, and comorbidities that preclude safe colonoscopy (23). Others have suggested that noncompliance may be due to a combination of factors at the patient, provider, and health systems levels (24). Stock et al (25) showed that a notification sent directly to FOBT-positive screening patients increased colonoscopy uptake. A telephone call reminder, in addition to a mailed notification, may also improve the acceptance rate of colonoscopy in patients with a positive FIT (26).
Our study had several limitations. We conducted the study in 3 Southwest tribal communities, so results are not generalizable to all AI/AN populations. CHRs were unable to carry out all outreach as directed by the study protocol, compromising the comparison in FIT return between groups 2 and 3. Finally, we cannot conclude that group 3 participants who returned FIT kits after the outreach did so as a proximal result of outreach instead of the mailing itself.

The elimination of structural barriers through direct mailing of FIT kits to eligible community members is a useful, population-based approach to increase CRC screening among AI/AN people. The role of CHRs in improving CRC screening efforts could be studied further. Identifying interventions that increase the use of FOBT or FIT among AI/AN populations could have important implications for the uptake of CRC screening services and for decreased CRC mortality.

\section{Acknowledgments}

We acknowledge community health representatives, laboratory staff, and all others from the 3 participating Tribal health care facilities for their dedication to this project throughout the study period and afterwards. Funding for this study came from the Division of Cancer Prevention and Control, Centers for Disease Control and Prevention (CDC). The findings and conclusions in this report are those of the authors and do not necessarily represent the official position of CDC. Use of trade names is for identification only and does not imply endorsement by the US Department of Health and Human Services. No copyrighted materials were used in this article.

\section{Author Information}

Corresponding Author: Donald Haverkamp, MPH, 1720 Louisiana Blvd, NE, No. 208, Albuquerque, NM 87110. Telephone: 505235-1163. Email: CYQ1@cdc.gov.

Author Affiliations: ${ }^{1}$ Centers for Disease Control and Prevention, Division of Cancer Prevention and Control, Albuquerque, New Mexico. ${ }^{2}$ Albuquerque Area Indian Health Board, Albuquerque, New Mexico.

\section{References}

1. White MC, Espey DK, Swan J, Wiggins CL, Eheman C, Kaur JS. Disparities in cancer mortality and incidence among American Indians and Alaska Natives in the United States. Am J Public Health 2014;104(Suppl 3):S377-87.

The opinions expressed by authors contributing to this journal do not necessarily reflect the opinions of the U.S. Department of Health and Human Services, the Public Health Service, the Centers for Disease Control and Prevention, or the authors' affiliated institutions. 
2. White A, Thompson TD, White MC, Sabatino SA, de Moor J, Doria-Rose PV, et al. Cancer screening test use - United States, 2015. MMWR Morb Mortal Wkly Rep 2017; 66(8):201-6.

3. Bibbins-Domingo K, Grossman DC, Curry SJ, Davidson KW, Epling JW Jr, García FAR, et al.; US Preventive Services Task Force. Screening for colorectal cancer: US Preventive Services Task Force recommendation statement. JAMA 2016; 315(23):2564-75.

4. Kanekar S, Petereit D. Walking forward: a program designed to lower cancer mortality rates among American Indians in western South Dakota. S D Med 2009;62(4):151-3,155-7,159.

5. Baron RC, Rimer BK, Breslow RA, Coates RJ, Kerner J, Melillo S, et al.; Task Force on Community Preventive Services. Client-directed interventions to increase community demand for breast, cervical, and colorectal cancer screening a systematic review. Am J Prev Med 2008;35(1Suppl):S34-55.

6. Sabatino SA, Lawrence B, Elder R, Mercer SL, Wilson KM, DeVinney B, et al.; Community Preventive Services Task Force. Effectiveness of interventions to increase screening for breast, cervical, and colorectal cancers: nine updated systematic reviews for the Guide to Community Preventive Services. Am J Prev Med 2012;43(1):97-118.

7. Walsh JM, Salazar R, Nguyen TT, Kaplan C, Nguyen LK, Hwang J, et al. Healthy colon, healthy life: a novel colorectal cancer screening intervention. Am J Prev Med 2010; 39(1):1-14.

8. Coronado GD, Golovaty I, Longton G, Levy L, Jimenez R. Effectiveness of a clinic-based colorectal cancer screening promotion program for underserved Hispanics. Cancer 2011; 117(8):1745-54.

9. Church TR, Yeazel MW, Jones RM, Kochevar LK, Watt GD, Mongin SJ, et al. A randomized trial of direct mailing of fecal occult blood tests to increase colorectal cancer screening. J Natl Cancer Inst 2004;96(10):770-80.

10. Myers RE, Sifri R, Hyslop T, Rosenthal M, Vernon SW, Cocroft $\mathrm{J}$, et al. A randomized controlled trial of the impact of targeted and tailored interventions on colorectal cancer screening. Cancer 2007;110(9):2083-91.

11. Indian Health Service. Resource and patient management system. https://www.ihs.gov/rpms/. Accessed May 14, 2019.

12. US Preventive Services Task Force. Screening for colorectal cancer: US Preventive Services Task Force recommendation statement. Ann Intern Med 2008;149(9):627-37.

13. Levy BT, Daly JM, Xu Y, Ely JW. Mailed fecal immunochemical tests plus educational materials to improve colon cancer screening rates in Iowa Research Network (IRENE) practices. J Am Board Fam Med 2012;25(1):73-82.
14. Jean-Jacques M, Kaleba EO, Gatta JL, Gracia G, Ryan ER, Choucair BN. Program to improve colorectal cancer screening in a low-income, racially diverse population: a randomized controlled trial. Ann Fam Med 2012;10(5):412-7.

15. Lasser KE, Murillo J, Medlin E, Lisboa S, Valley-Shah L, Fletcher RH, et al. A multilevel intervention to promote colorectal cancer screening among community health center patients: results of a pilot study. BMC Fam Pract 2009; 10(1):37.

16. Redwood D, Provost E, Lopez ED, Skewes M, Johnson R, Christensen $\mathrm{C}$, et al. A process evaluation of the Alaska Native Colorectal Cancer Family Outreach Program. Health Educ Behav 2016;43(1):35-42.

17. Coronado GD, Schneider JL, Sanchez JJ, Petrik AF, Green B. Reasons for non-response to a direct-mailed FIT kit program: lessons learned from a pragmatic colorectal-cancer screening study in a federally sponsored health center. Transl Behav Med 2015;5(1):60-7.

18. Coronado GD, Sanchez J, Petrik A, Kapka T, DeVoe J, Green B. Advantages of wordless instructions on how to complete a fecal immunochemical test: lessons from patient advisory council members of a federally qualified health center. $\mathrm{J}$ Cancer Educ 2014;29(1):86-90.

19. Kadiyala S, Strumpf EC. Are United States and Canadian cancer screening rates consistent with guideline information regarding the age of screening initiation? Int J Qual Health Care 2011;23(6):611-20.

20. Wernli KJ, Hubbard RA, Johnson E, Chubak J, Kamineni A, Green BB, et al. Patterns of colorectal cancer screening uptake in newly eligible men and women. Cancer Epidemiol Biomarkers Prev 2014;23(7):1230-7.

21. Perdue DG, Haverkamp D, Perkins C, Daley CM, Provost E. Geographic variation in colorectal cancer incidence and mortality, age of onset, and stage at diagnosis among American Indian and Alaska Native people, 1990-2009. Am J Public Health 2014;104(Suppl 3):S404-14.

22. Hubbard RA, Johnson E, Hsia R, Rutter CM. The cumulative risk of false-positive fecal occult blood test after 10 years of colorectal cancer screening. Cancer Epidemiol Biomarkers Prev 2013;22(9):1612-9.

23. Gupta S, Halm EA, Rockey DC, Hammons M, Koch M, Carter $\mathrm{E}$, et al. Comparative effectiveness of fecal immunochemical test outreach, colonoscopy outreach, and usual care for boosting colorectal cancer screening among the underserved: a randomized clinical trial. JAMA Intern Med 2013; 173(18):1725-32.

24. Martin J, Halm EA, Tiro JA, Merchant Z, Balasubramanian BA, McCallister K, et al. Reasons for lack of diagnostic colonoscopy after positive result on fecal immunochemical test in a safety-net health system. Am J Med 2017;130(1):93.e1-7.

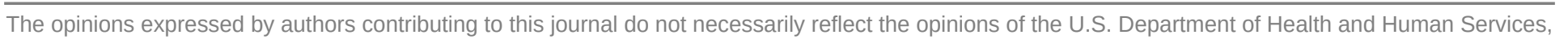
the Public Health Service, the Centers for Disease Control and Prevention, or the authors' affiliated institutions. 
25. Stock D, Rabeneck L, Baxter NN, Paszat LF, Sutradhar R, Yun $\mathrm{L}$, et al. Mailed participant reminders are associated with improved colonoscopy uptake after a positive FOBT result in Ontario's ColonCancerCheck program. Implement Sci 2015; 10(1):35.

26. Cha JM, Lee JI, Joo KR, Shin HP, Park JJ. Telephone reminder call in addition to mailing notification improved the acceptance rate of colonoscopy in patients with a positive fecal immunochemical test. Dig Dis Sci 2011;56(11):3137-42. 


\section{Table}

Table. Colorectal Cancer Screening Interventions in 3 Tribally Operated Health Care Centers Using the Fecal Immunochemical Test (FIT), 3 Intervention Groups, April-November $2014^{\mathrm{a}}$

\begin{tabular}{|c|c|c|c|c|}
\hline Variable & $\begin{array}{c}\text { Group 1, Usual Care } \\
(n=566)\end{array}$ & $\begin{array}{l}\text { Group 2, Mailing Alone }{ }^{c} \\
(n=361)\end{array}$ & $\begin{array}{l}\text { Group 3, Mailing + } \text { Outreach }^{d} \\
(n=361)\end{array}$ & Total $(\mathrm{N}=1,288)$ \\
\hline \multicolumn{5}{|l|}{ Center } \\
\hline 1 & $257(45.4)$ & $133(36.8)$ & $133(36.8)$ & $523(40.6)$ \\
\hline 2 & $95(16.8)$ & $95(26.3)$ & $95(26.3)$ & $285(22.1)$ \\
\hline 3 & $214(37.8)$ & $133(36.8)$ & $133(36.8)$ & $480(37.3)$ \\
\hline \multicolumn{5}{|l|}{ Age, $y^{e}$} \\
\hline Mean, (standard deviation) & $60.6(7.0)$ & $60.8(6.8)$ & $59.8(6.7)$ & $60.4(6.9)$ \\
\hline $50-59$ & $284(50.3)$ & $170(47.2)$ & $194(53.7)$ & $648(50.4)$ \\
\hline $60-69$ & $204(36.1)$ & $149(41.4)$ & $131(36.3)$ & $484(37.6)$ \\
\hline $70-75$ & $77(13.6)$ & $41(11.4)$ & $36(10.0)$ & $154(12.0)$ \\
\hline Women & $291(51.4)$ & $179(49.6)$ & $200(55.4)$ & $670(52.0)$ \\
\hline
\end{tabular}

${ }^{a}$ Values are number (percentage) unless otherwise indicated.

${ }^{\mathrm{b}}$ No outreach apart from provider screening advice given during clinic visits.

${ }^{\mathrm{c}}$ Mailing FIT kit with instructions for use.

${ }^{\mathrm{d}}$ Mailing FIT kit with instructions for use. If no response, follow-up telephone call after 4 weeks, follow-up home visit after 8 weeks, and telephone call after 12 weeks.

${ }^{\mathrm{e}}$ Values for 3 groups may not equal totals because some participants did not provide age. 